\title{
LE JOUR DE RÉCEPTION EN TURQUIE URBAINE. RÉSEAUX SOCIAUX ET SOCIABILITÉ
}

\author{
Hélène Bayard-Çan
}

Presses Universitaires de France | Ethnologie française »

2014/2 Vol. 44 | pages 279 à 288

ISSN 0046-2616

ISBN 9782130628927

Article disponible en ligne à l'adresse :

https://www.cairn.info/revue-ethnologie-francaise-2014-2-page-279.htm

Distribution électronique Cairn.info pour Presses Universitaires de France.

(C) Presses Universitaires de France. Tous droits réservés pour tous pays.

La reproduction ou représentation de cet article, notamment par photocopie, n'est autorisée que dans les limites des conditions générales d'utilisation du site ou, le cas échéant, des conditions générales de la licence souscrite par votre établissement. Toute autre reproduction ou représentation, en tout ou partie, sous quelque forme et de quelque manière que ce soit, est interdite sauf accord préalable et écrit de l'éditeur, en dehors des cas prévus par la législation en vigueur en France. Il est précisé que son stockage dans une base de données est également interdit. 
Hélène Bayard-Çan

\title{
Le jour de réception en Turquie urbaine. Réseaux sociaux et sociabilité
}

Université de Çukurova

\author{
RÉSUMÉ \\ Hélène Bayard-Çan \\ Çukurova Üniversitesi Eğitim Fakültesi \\ Fransız Dili Eğitimi ABD \\ 01330 Balcalı Sarıçam / Adana \\ Turquie \\ hbayardcan@cu.edu.tr
}

Si les visites occupent une place essentielle dans la sociabilité turque, les jours de réception, les gün, tiennent lieu d'institution. L'article examine, à partir d'enquêtes et d'observations conduites dans plusieurs villes de Turquie, les caractéristiques de ce mode de sociabilité. Après un examen de ses dimensions sociale, psychologique, économique et religieuse, il aborde le gün d'un point de vue réticulaire et démontre son importance dans la constitution, l'extension et la mobilisation du réseau social de chacun. Mots-clés : Sociabilité. Gün. Jour de réception. Réseau social. Turquie.

Les visites occupent une place essentielle dans la sociabilité en Turquie. Elles peuvent être le fait d'une invitation à prendre le thé ou à dîner, notamment au moment du ramadan pour le repas de rupture du jeûne (iftar). On peut aussi inviter à prendre un thé ou un café un voisin rencontré par hasard ou qui a sonné à la porte. Mais plus qu'inviter, c'est rendre visite qui importe. Le terme de davetli, littéralement l'" invité ", est d'ailleurs peu employé, au profit du terme de misafir, le visiteur ${ }^{1}$. Rendre visite (ziyaret etmek) est en effet un honneur que l'on rend. Certaines visites prennent même un caractère obligatoire, en s'inscrivant dans un cadre de droits et devoirs, afin d'engager ou de maintenir des relations de sociabilité entre parents, amis, voisins. Elles ont ainsi lieu pour célébrer des fêtes religieuses (les visites de bayram de la fin du ramadan ou de la fête du sacrifice), pour souhaiter la bienvenue à quelqu'un qui vient d'emménager ou qui revient d'un long voyage, du service militaire ou de pélerinage, souhaiter un bon rétablissement à quelqu'un de malade, apporter ses condoléances à la famille d'un défunt, fêter un événement important comme un mariage, une naissance, voire une transformation dans le logement, etc. D'autres visites sont rendues de façon quotidienne et informelle ${ }^{2}$. Tout comme le don décrit par Marcel Mauss [1993], la visite répond aux obligations de donner, recevoir et rendre : obligation de rendre visite dans certaines occasions prédéfinies, obligation de recevoir le visiteur qu'il n'est pas convenable de renvoyer et dont on ne peut refuser la venue, et obligation de rendre cette visite si l'on veut que les relations perdurent.

Dans ce cadre, le jour de réception ou gün (littéralement "jour») s'oppose aux visites occasionnelles ou fortuites. Sorte de tontine fondée sur un système de cooptation de ses membres [Sauner, 2001], il s'agit d'une réunion, le plus souvent entre femmes, organisée de façon périodique chez chaque participante, à tour de rôle ${ }^{3}$. C'est, avec les visites aux parents et voisins, une des principales activités de loisir des femmes dans les petites agglomérations [Sönmez et al., 2010 : 96]. Formalisé, le gün répond à des principes précis et s'inscrit dans la régularité : régularité des participants, de la fréquence, du jour et de l'heure, et même des mets et boissons offerts (ikram).

Cet article s'attache à déterminer les multiples aspects de ce qui est une institution en Turquie urbaine. Si les 
dimensions sociales [Aswad, 1974 ; Benedict, 1974 ; Ekal, 2006 ; Kiray, 1981 ; Sönmez et al., 2010 ; Tapper, 1990], économiques [Khatib-Chahidi, 1995], psychologiques [Sauner, 2001 ; Wolbert, 1992, 1996] ou religieuses sont pour la plupart largement documentées, l'aspect réticulaire, c'est-à-dire la place du réseau, pourtant déterminant de ce mode de sociabilité, n'a été qu'évoquée [Sauner, 2001]. Après avoir présenté les grandes caractéristiques du gün, l'analyse approfondira la question de l'organisation réticulaire : son mode de constitution et son rôle dans la dynamique sociale globale des participants ${ }^{4}$.

\section{- Du jour de réception au gün : principes d'un mode de sociabilité}

Le gün ${ }^{5}$ est la continuité de ce qui s'appelait encore dans les années 1970 le "jour de réception » (kabul günü), au cour duquel les femmes des classes sociales moyennes ou supérieures décidaient d'ouvrir les portes de leurs salons et de recevoir celles qui le désiraient [Benedict, 1974 ; Aswad, 1974]. Cette forme de sociabilité a aujourd'hui glissé de la couche aisée de la population vers la couche moyenne, un peu partout en Turquie [Wolbert, 1996]. À l'inverse des jours de réception originels accueillant toutes les visiteuses qui le souhaitent tout au long de la journée, les gün actuels ne durent que quelques heures préalablement déterminées.

Les gün se présentent sous diverses formes et rassemblent de trois-quatre à une vingtaine de femmes, composant un groupe fixe qui se retrouve chez une de ses participantes, à tour de rôle et à intervalles réguliers (variant d'une fois par semaine à une ou deux fois par mois). On trouve aujourd'hui plusieurs sortes de gün: kahve günü (jour du café), para günü (jour de l'argent) ou paralı gün (jour avec de l'argent) ou autrement dit altın günü (jour de l'or), dolar günü (jour du dollar) ou euro günü (jour de l'euro), voire simplement gün (jour). Ces noms mettent l'accent sur l'aspect spécifique d'une telle réunion. Si, dans le premier cas, est mis en avant le café offert aux visiteuses, les autres soulignent une composante essentielle de la réunion qui consiste à récolter auprès de chaque participante de l'argent destiné à l'hôtesse. Le type du don, ainsi que son montant, est déterminé à l'avance, lors de l'organisation du cycle des visites.

Ces réunions se tiennent essentiellement dans la journée, alors que les hommes sont au travail, la maison étant libre et la femme déchargée des obligations concernant son mari ou ses enfants scolarisés. Par ailleurs, c'est un regroupement social dont l'homme est généralement exclu; il s'agit d'une affaire de femmes et, si le mari de l'hôtesse se trouve à la maison, il s'efforce d'être le plus discret possible et n'apparaît pas tant que dure la réunion. On retrouve là un trait important des relations sociales traditionnelles en Turquie, qui consiste à séparer les sexes, même s'il existe, bien que plus rarement, des gün mixtes, en soirée.

\section{- Un gïn à Adana chez Rukiye}

J'ai eu l'occasion d'assister à différents cycles de gün durant la période 2001-2002 organisés entre plusieurs voisines d'un quartier de classe moyenne d'Adana. L'idée d'une réunion était venue à deux voisines qui en ont fait part à des voisines d'un autre immeuble. Elles tenaient à partager un gün qui leur permettrait de se retrouver de façon régulière, se plaignant de ce que, depuis un an, leur gün commun ayant cessé, elles n'avaient plus l'occasion de se réunir. Durant cette période, trois séries de gün ont ainsi été organisées autour de ce noyau dur de voisines. La douzaine de participantes, âgées de 30 à 56 ans, pouvait varier d'une série à l'autre, certaines quittant le gün, par manque de disposition personnelle ou parce qu'elles n'avaient pas pu être assidues lors de la précédente série, d'autres s'y insérant. Le gün regroupait exclusivement des femmes au foyer habitant une même résidence ou des membres de leur parenté. Leurs maris étaient commerçants, artisans ou retraités. L'une d'entre elles était une institutrice en retraite. Le gün avait lieu régulièrement un matin par semaine du mois de septembre au mois de mai, mois où traditionnellement les gün s'arrêtent en raison du début des départs en vacances.

La première réunion devait avoir lieu chez Rukiye qui s'était proposée pour recevoir. Halide est venue me chercher, en compagnie d'Ayşe, une voisine avec laquelle elle entretenait d'intenses relations. Celle-ci était accompagnée de sa petite-fille de quelques mois. Nous sommes arrivées vers $10 \mathrm{~h} 30$ puis, peu après nous, est venue Bilge, une voisine du quatrième étage, suivie de la fille d'Ayşe, qui habitait dans un immeuble voisin, avec sa fille de 5 ans et sa cousine Ayşegül résidente elle aussi d'un immeuble voisin. Ensuite Dilek, du même immeuble que Rukiye, est venue avec sa belle-fille et enfin, la belle-mère de la fille d'Halide. Plusieurs personnes participant aussi à ce gün, 
n'avaient cependant pas pu être présentes ce jour-là : la belle-sœur de la fille d'Ayşe, ainsi que la belle-fille d'Halide, habitant elle aussi dans la résidence mais qui, en raison de son activité professionnelle, ne participait au gün que pour verser et recevoir l'argent quand son tour viendrait. Une parente de Rukiye, de passage à Adana ce jour-là, s'était jointe au groupe. Au début du cycle, les femmes avaient décidé que, contrairement à ce qui se fait dans la plupart des gün, où l'hôtesse offre à ses invitées de nombreux plats, elles se contenteraient d'offrir un café, pour des motifs de santé (ne pas prendre de poids) et pour ne pas occasionner de frais à l'hôtesse en ces temps de crise économique.

À son arrivée, toute nouvelle venue saluait chaque visiteuse en l'embrassant, puis allait s'asseoir, les plus jeunes cédant leur place sur un canapé ou dans un fauteuil aux personnes les plus âgées pour aller s'asseoir sur une chaise. Les sièges étaient arrangés en cercle, de façon à ce qu'aucune ne tourne le dos aux autres. Chacune demandait des nouvelles à chaque participante. Au fur et à mesure des arrivées, l'hôtesse préparait et servait les cafés turcs. Ayşegül, parmi les plus jeunes du groupe (la trentaine), aidait pour le service, en commençant par les femmes plus âgées. Sur la table, dans des coupes, se trouvaient des paquets de cigarettes de différentes marques, présents offerts aux visiteuses. Les conversations se déroulaient généralement entre deux ou trois personnes, parfois, entre tous les membres du groupe. Lors de ce premier gün, l'état de santé des unes et des autres a tout d'abord été évoqué. Ce jour-là, plusieurs personnes ayant mal à la tête, Rukiye a sorti son tensiomètre et Ayşegül a mesuré la tension de celles qui le désiraient, ce qui a provoqué de nombreux commentaires. À la suite de cris d'enfants dans l'immeuble, Rukiye s'est plainte des bruits incessants des enfants de sa voisine, entraînant la conversation sur la bienséance du voisinage. À plusieurs reprises, des voisines ont commenté la nouvelle coloration des cheveux d'Halide. Ensuite, la conversation a porté sur le cours des tomates et les lieux où se les procurer au meilleur prix. Parmi d'autres conversations, Dilek a parlé de la préparation du mariage de son fils en novembre et Halide lui a proposé son aide. Ayşe, quant à elle, a profité de ce que toutes les voisines fussent réunies pour leur faire part de la cérémonie qu'elle allait organiser le vendredi suivant pour l'anniversaire de la mort de son mari et y a convié chaque personne individuellement. Lors de ce premier gün, Halide avait apporté son tricot. Rukiye a évoqué le cours de couture qu'elle suivait, puis, cédant à l'insistance de ses amies, a apporté son ouvrage que toutes les visiteuses ont examiné et commenté, l'une d'elle prodiguant ses conseils.

Il avait été décidé, pour cette série de gün, d'apporter à l'hôtesse une pièce d'or de taille moyenne (un "demi-or", yarım altın). Le montant de la pièce (47 $000000 \mathrm{TL}$, soit l'équivalent d'environ $37 €$ selon le cours du change de l'époque) devait être divisé par le nombre de personnes cotisant. De nombreuses palabres ont été nécessaires pour déterminer le nombre de participantes et effectuer les calculs, la principale difficulté étant de déterminer qui exactement participait au gün, en tenant compte des personnes absentes mais en excluant l'invitée de Rukiye qui n'était là que ponctuellement. Certaines ont payé à la place des absentes. Le ramassage de l'argent et surtout le décompte ont donné lieu à de nouvelles discussions, certaines se demandant si l'hôtesse devait aussi participer, une autre refusant catégoriquement, dans un souci d'égalité vis-à-vis des fois suivantes, que l'on arrondisse le montant versé par chacune. Une autre, enfin, chargée de la collecte, dut refaire les décomptes plusieurs fois et s'assurer que chacune avait bien versé sa part ou celle des absentes. Selon la décision préalablement adoptée, toutes les participantes étaient parties à midi, chacune s'en allant avec celle qui l'avait accompagnée.

\section{- Le gün, un mode de sociabilité et un outil de prestige}

À l'instar du jour de réception, le gün est avant tout un lieu de sociabilité auquel est systématiquement associée la notion de commérages (dedikodu) dont il est souvent le cadre. Les conversations débutent systématiquement par l'échange de nouvelles entre toutes les participantes avant de s'engager vers d'autres sujets portant notamment sur les préoccupations quotidiennes ou sur la vie des connaissances communes et du quartier. Berna Ekal [2006] note que c'est ainsi un important lieu de transmission des normes sociales. Elle donne l'exemple des femmes ayant migré des campagnes vers les villes qui apprennent, grâce au gün, à se conformer aux normes de la vie moderne à travers le comportement idéal que doit adopter une belle-mère.

Parallèlement aux discussions, la commensalité se trouve au centre de la réunion. Ce qui est offert aux invitées $\left(\right.$ ikram $\left.^{6}\right)$ est déterminé en début de cycle, règle à laquelle chacune devra se conformer, sous peine de 
protestations ou de commérages. Cela va donc d'un simple café à de multiples mets :

L'année dernière, on proposait beaucoup de choses à manger, c'était fatigant. Et puis nous ne voulons pas manger, de peur de prendre des kilos. Alors cette année on a posé une condition : soit on va faire un plat à base de boulgour soit ce sera un mets salé et un sucré (bir tatl bir tuzlu) et rien d'autre à part ça.

Quel que soit l'ikram, un café est toujours offert à la visiteuse peu après son arrivée, boisson de la sociabilité par excellence. Un proverbe ne dit-il pas qu'" on se souvient pendant quarante ans d'une tasse de café " (bir fincan kahvenin kırk yul hatır vardir), montrant ainsi son importance dans la culture turque.

Lieu de sociabilité, le gün est aussi enjeu de prestige. Si ce point a été largement démontré pour le kabul günü [Benedict, 1974 ; Aswad, 1974 ; Tapper, 1990] au cours duquel la présence de femmes appartenant aux hautes classes de la société permet non seulement de révéler le statut de l'hôtesse, mais aussi de l'accroître, il en va de même, dans une moindre mesure, du gün des classes moyennes. Celui-ci est généralement un lieu de représentation de soi. Hormis lorsqu'il est organisé en groupe restreint entre personnes intimes, chacune s'attache à soigner sa tenue. Si, lors de visites informelles, on peut s'asseoir dans le petit salon, lieu de séjour de la famille au quotidien, le gün, lui, a lieu dans le salon qui est une pièce "acquise à l'apparat où est signifié le statut de la maison, et par là à l'événement " [Fliche, 2006: 54]. Les ikram font partie intégrante de cette représentation, c'est pourquoi le gün rime souvent avec une multitude de mets qui seront par la suite commentés par les visiteuses après leur départ. Chacune doit se surpasser, et faire preuve d'originalité dans ses talents de cuisinière, en préparant ce qui est à la mode au niveau culinaire. Il s'agit parfois d'une véritable compétition pour être celle qui offrira le plus de plats. Certaines insistent donc pour que la consigne donnée au départ à ce sujet soit respectée. Se surpasser dans les ikram ajoute au prestige individuel. La règle communément admise de "deux mets salés, deux sucrés » (iki tuzlu iki tatli), décrétée au début du cycle du gün, n'est plus respectée et le nombre de plats augmente, chacune voulant être à la hauteur ou dépasser les performances des précédentes. Souvent, le rappel à l'ordre est nécessaire pour arrêter l'escalade.

$\mathrm{Si}$, dans certaines réunions, la présentation vestimentaire ainsi que la compétition alimentaire ont disparu, le prestige et le niveau social semblent s'afficher d'une autre façon. La présence au gün joue toujours un rôle impor- tant et la maitresse de maison se sent humiliée lorsque ses invitées sont en retard ou absentes sans raison explicite: "Deux personnes ne sont pas venues chez moi, eh bien moi je leur ai dit que je n'irai pas chez elles. " On retrouve ici tout le sens de la notion d' "honorer" quelqu'un par sa visite car le nombre de visiteurs indique le niveau de prestige de l'hôte' ${ }^{7}$ D'autre part, le statut social se révèle aussi directement par le fait d'assister ou non à un gün et selon le nombre de gün auxquels on peut participer, indice du niveau de richesse. En effet, la composante économique du gün permet de discerner les personnes matériellement capables de participer à de telles réunions. Le nombre de gün auxquels on se joint et la participation financière versée à chacun d'eux dépendent de ses possibilités économiques, et participer à de nombreux gün permet d'afficher son capital social comme son capital économique. Le gün est le mode de sociabilité par excellence des femmes au foyer :

En général, avant les femmes ne travaillaient pas. Et une personne qui ne travaille pas, que fait-elle ? Elle voit forcément plus les autres personnes, elle participe à des réunions, elle fait un gün, elle fait un "jour de l'or ", elle fait je ne sais pas quel gün, elles se retrouvent quoi. Un jour chez moi, un jour chez vous, un jour chez l'autre, elles se réunissent chaque jour. Les personnes qui travaillent, quand bien même elles le voudraient, elles ne le peuvent pas (Nuray, 59 ans, commerçante).

Le gün donne l'opportunité de partager des mêmes préoccupations. C'est pourquoi de nombreux échanges tournent autour des tâches ménagères. Aussi, au moment de se retrouver, les participantes commencent par parler de ce qu'elles ont fait dans la matinée ou projettent de faire, comme nettoyer les tapis ou les rideaux. De nombreuses discussions portent sur les prix des biens, notamment sur ceux des fruits et légumes, la présence de promotions à tel endroit. De plus, les réunions féminines sont le lieu d'échanges de conseils concernant la façon d'effectuer un tricot ou de la dentelle, activités aussi régulièrement pratiquées durant la réunion où chacune admire le travail des autres. D'après Sönmez et al. [2010], il arrive que les femmes jouent d'un instrument de musique ou réalisent de la peinture sur bois. Lors d'un des gün auxquels j'ai assisté, nous avions pendant plusieurs réunions effectué ensemble des raviolis pour notre hôtesse, jusqu'à ce que nous abandonnions, car certaines s'impliquaient moins que d'autres dans la tâche. Les réunions sont en outre le lieu privilégié pour l'échange de recettes de cuisine ou bien encore de suggestions de menus pour le soir. De plus, les participantes 
$\mathrm{y}$ emmènent facilement leurs filles et belles-filles, faisant du gün un lieu d'apprentissage de la sociabilité et de certains savoir-faire propres au rôle féminin.

\section{- Les diverses dimensions du gün}

\section{- La dimension économique}

L'aspect économique du gün d'aujourd'hui n'existait pas dans le kabul günü. La collecte d'argent destiné à l'hôtesse est désormais une composante essentielle de la réunion, au point de lui donner, on l'a vu, son nom. Le prétexte économique enrichit les fonctions de la réunion, car il nécessite sérieux et formalisation : le gün est prétexte à se cotiser et faire de petites économies. La participation à un gün engage les participantes à y consacrer assidûment une certaine somme d'argent, discipline à laquelle il est difficile de se tenir en temps normal. "En fait, c'est pour nous un moyen de faire des économies. C'est une économie obligatoire qui ne grève pas trop notre budget. " L'aspect économique en est parfois même la composante exclusive, certaines personnes ne pouvant participer que financièrement ; c'est notamment le cas quand des collègues organisent un gün sans réunion ou quand des personnes qui habitent loin (souvent des membres de la famille de participantes) ou travaillent, se contentent d'envoyer régulièrement au gün l'argent nécessaire. Leur part est généralement récoltée en fin de cycle, pendant l'été, quand les réunions ont cessé ou lors du mois du ramadan qui ouvre une parenthèse dans ces réunions peu compatibles avec le jeûne. La dimension économique donne lieu à de nombreuses discussions, et une partie de la réunion se passe à effectuer les calculs et à récolter l'argent. Plus que l'aspect financier, le souci d'égalité prédomine, chacune devant payer à égalité, tout comme on retrouve ce souci d'égalité dans le nombre de plats proposés, trop en faire étant une façon de provoquer un sentiment d'infériorité parmi les autres participantes.

Le montant du don est variable d'un gün à l'autre. Halide (56 ans, femme au foyer), que j'interrogeais sur son expérience des gün, se souvenait que dans les années 1960 elle participait à des "jours de l'argent " (gümüşs günü) où l'on apportait à l'hôtesse un certain grammage du métal précieux. Cette pratique a aujourd'hui disparu pour laisser la place à l'or ou à des devises, euros ou dollars, suivant le cours du marché et la confiance accordée à ces différentes valeurs ${ }^{8}$. Le montant est déterminé à l'avance par toutes les participantes, suivant la situation économique de chacune. Il peut s'agir de donner 10 dollars par personne, de quoi acheter une pièce d'or de taille variable, une "pièce entière " ( tüm altın dite aussi Cumhuriyet, "République", et correspondant à 7 grammes d'or), ou des pièces plus petites de la moitié (yarm altın) ou du quart (çeyrek altın) de la valeur de la première, des euros (avant l'apparition de l'euro, il s'agissait de marks allemands) ou des dollars. Un groupe de commerçants apportait ainsi mensuellement à son hôte un total de 1000 euros. Dans d'autres milieux, la réunion sert à se procurer certains biens, tout le monde se cotisant pour acheter à l'hôtesse une friteuse, une cocotte-minute, un robot de cuisine, un mixer, une housse de couette, une serviette ou tout autre article déterminé par le groupe. Dans les gün les plus modestes, il peut aussi s'agir de produits de première nécessité, comme de la farine ou du sucre.

Que devient l'argent du gün? Les pièces d'or peuvent être utilisées telles quelles, quand s'agit de faire un cadeau lors d'un mariage ou d'une naissance. L'argent mis de côté au fil de réunions sur plusieurs années peut servir pour des dépenses exceptionnelles (achat de produits électroménagers). Halide l'utilise pour financer une partie de ses vacances, Rukiye (51 ans, femme au foyer), tout comme plusieurs amies d'Halide dont la situation économique était plus aisée, s'était acheté un bracelet en or.

\section{- La dimension psychologique}

Au-delà de la simple sociabilité, ces réunions féminines ont une composante psychologique. Barbara Wolbert [1996] constate que, si le gün est avant tout moyen de se distraire et un lieu de partage, il permet en outre d'effectuer une sorte de thérapie de groupe. Elle montre, avec Marie-Hélène Sauner [2001], le rôle de cette institution dans la réintégration des femmes de retour d'immigration après de nombreuses années passées en Allemagne, et notamment comment le gün leur a permis de quitter leur statut d'almanyalı pour devenir turques à part entière'. Les jours de réception, sans hommes et réunissant des femmes qui se sont choisies et ont appris à se connaitre, favorisent le partage d'une certaine intimité. Les unes et les autres peuvent faire part de leurs problèmes ou s'apporter un soutien mutuel, comme ce fut le cas lorsque l'une des participantes subit un avortement, ce qui offrit aussi l'occasion de débattre du sujet. C'est en effet un lieu de partage où l'on évoque avec les autres des moments 
difficiles, dans une certaine communion. Ainsi, Rukiye, l'une des participantes à un gün entre voisines, qui venait de perdre son fils d'une vingtaine d'années dans un accident de voiture, a été soutenue par toutes les participantes au fil des différentes réunions. Un jour, nous avions profité du gün pour nous rendre à nouveau chez Rukiye dont la fille, cette fois, venait de faire une tentative de suicide. Notre visite avait donc pour but, outre de participer au jour de réception, de souhaiter un bon rétablissement à Ebru et d'apporter notre soutien à Rukiye dans ce moment difficile. Lors de notre venue, nous n'avons pas manqué de rappeler l'accident de son fils. Et, dans un grand moment d'émotion, chacune en était venue à penser à ses propres morts et à évoquer ses douloureux souvenirs. En fin de visite, plusieurs voisines s'étaient ainsi mises à pleurer et tout le monde partageait le même sentiment de tristesse.

\section{- L'aspect religieux}

Les réunions peuvent aussi comporter un volet spirituel. En effet, il n'est pas rare que la conversation porte sur la pratique religieuse, notamment sur ce qui est permis ou non par l'islam ou selon tel hoca ${ }^{10}$ diffusé à la télévision. Bien que pour certaines cela ne soit pas convenable, le gün peut être l'occasion d'organiser une cérémonie religieuse, ou une lecture du Coran, comme pour cette femme initiatrice d'une cérémonie votive avec préparation de helva ${ }^{11}$ pour remercier Allah de la naissance de son enfant. Depuis quelques années, des femmes voilées se rendent avec une des participantes à ces réunions et y lisent un extrait du Coran qu'elles commentent. Selon mes informatrices, elles seraient rémunérées par des organismes religieux et peut-être même soutenues par le gouvernement islamiste afin de faire du prosélytisme, ce qui n'est pas toujours du goût de toutes. J'avais assisté à une telle intervention et, lorsque la femme fut partie, plusieurs femmes, qui par politesse n'avaient pas osé interrompre la discussion religieuse, ont menacé de ne plus participer au gün si cela devait se reproduire : elles venaient pour rencontrer leurs voisines et non pour recevoir une leçon de religion.

\section{- Le jour de réception au sein du réseau}

Outre ses nombreuses dimensions fonctionnelles, le gün peut être organisé en réseau. Il rassemble essentiellement des personnes appartenant à un même cercle social. Lorsque l'on analyse des réseaux, on appelle "cercle social» un ensemble d'individus conscients d'appartenir à un même groupe social, partageant des valeurs communes et entre lesquels existent des interactions [Degenne et Forsé, 1994 : 216-217]. Tout individu est ainsi impliqué dans plusieurs cercles sociaux distincts et dans de multiples relations au sein de ces groupes. Les cercles sociaux les plus courants d'un individu sont les cercles de parenté, d'amitié, de voisinage, et de travail, qui comprend ses collègues [Wellman, 1979 ; Grossetti, 2005 : 292 ; Barnes, 1954 : 40]. À côté de ces groupes peuvent exister, pour chacun, de multiples cercles au sein desquels la personne interviendra. Tout comme les cercles sociaux sont à la base de la plupart des relations sociales [Grossetti, 2005], les gün démarrent fréquemment sous l'impulsion de plusieurs membres d'un même cercle social. On retrouve ainsi des jours de réception entre voisines, entre parentes, entre collègues... qui sont chacun indépendants et avec un mode de fonctionnement propre. Le gün est d'ailleurs souvent perçu comme l'une des activités majeures des relations de voisinage féminines [Bayard-Çan, 2008]. C'est aussi un moyen de se rassembler entre personnes exerçant une même activité : entre commerçants d'un mềme quartier, entre collègues. Les gün font ainsi apparaître clairement les frontières des cercles sociaux et la volonté de rester entre soi. Leur constitution se fonde sur le principe d'homophilie selon lequel les relations sociales ont lieu de préférence avec ses semblables, entraînant ainsi un cloisonnement des sphères sociales du réseau d'un individu. Les femmes font facilement partie de plusieurs gün, chacun regroupant les membres de différentes sphères sociales, ce qui permet de recréer un groupe et de renforcer la notion d'appartenance à un groupe constitué. Les jours de réception auxquels participe Halide sont à cet égard exemplaires :

\footnotetext{
Maintenant, [avec Ayşe ${ }^{12}$ ] nous avons trois jours de réception. Nous avons un gün tous les 15 jours avec des amies enseignantes, la cagnotte y est de 10 dollars. Et puis avec mes parentes nous en faisons un par mois, là, nous nous retrouvons à sept-huit parentes et chacune d'entre nous apporte un "quart d'or". Et puis nous avons le café du matin, entre voisines. Chaque semaine le prix d'une demi-pièce d'or est partagé entre toutes les participantes.
}

Le groupe commence souvent par un cercle social fermé, puis plusieurs personnes vont ensuite être cooptées et intégrées par les membres du groupe. Si les participantes parlent de "faire un gün » (bir gün yapmak) pour exprimer le fait d'y participer, elles utilisent aussi 
des expressions comme "entrer dans un gün" (bir güne girmek) ou "sortir d'un gün" (bir günden çıkmak). C'est ainsi un moyen d'intégrer les individus dans le cercle social concerné. Fermé au départ, le groupe présente une forte capacité d'intégration et joue à cet égard un important rôle dans la sociabilisation. C'est notamment le cas lors des réunions de voisines qui regroupent la plupart du temps des femmes au foyer et, s'ouvrant aux femmes qui travaillent, leur permettent de rester en contact régulier avec les voisines. Les gün sont souvent une première étape, nécessaire et suffisante, pour entretenir un minimum de relations de voisinage qui pourront s'intensifier par la suite, opportunité pour la nouvelle venue de trouver sa place au sein de l'immeuble et du réseau de voisines.

Les visites en groupe permettent ainsi de connaitre les autres voisines et, par les conversations et les commérages qui y ont cours, de repérer les codes communs. Cependant, malgré des rencontres chaleureuses et fréquentes avec les mêmes personnes, certaines de mes informatrices ignorent le nom de plusieurs membres de leur gün, ce qui montre que certaines relations restent encore superficielles. En raison de son offre d'intégration, le jour de réception est le moyen idéal pour étendre son réseau au-delà même du cercle social originaire, étoffer et diversifier son réseau social. En effet, l'intégration de membres se faisant de proche en proche, il n'est pas rare que des personnes étrangères au groupe initial viennent à la suite d'une participante se greffer au groupe, chacune pouvant y amener des personnes appartenant à ses différents cercles sociaux. Il s'agit habituellement d'une amie proche, une voisine ou un membre de sa famille. Halide raconte ainsi comment elle a été acceptée dans le gün entre institutrices auquel participait la belle-mère de sa fille et comment elle y a elle-même fait venir plus tard sa voisine et amie :

La belle-mère de ma fille est enseignante, elle m'a fait rencontrer ses amies. Moi, j'y suis allée une année ou deux de cette façon. Et l'année dernière je leur ai présenté Ayşe hanim, je leur ai dit que c'était ma voisine et leur ai demandé si elle pouvait participer. Elles ont répondu «bien sûr» et cette année deux autres amies ont amené leur voisine. C'est pour ça que nous étions neuf avec moi et cette année nous sommes douze. Donc au début il n'y avait que des enseignantes et maintenant il y a trois-quatre personnes de l'extérieur, qui sont femmes au foyer.

Ayşe (49 ans, femme au foyer) avait de même fait venir à son gün ses deux filles, l'une d'entre elles y ayant ainsi entraîné sa cousine et sa belle-sœur. Le réseau familial est donc très souvent mis à contribution pour " recruter " des participantes, ce qui permet d'étendre le réseau à la jeune génération. Tijen (38 ans, femme au foyer) avait ainsi fait la connaissance de bon nombre de voisines, du temps où elle accompagnait sa mère qui habitait dans la résidence. La nouvelle venue vient avec une participante et rencontre ainsi tous les membres du groupe avec lesquels elle finit par entretenir des liens d'amitié : "Nous avions un "jour du café " ensemble, c'est pourquoi nous nous connaissons. [...] Maintenant, moi j’y suis allée, elle est venue, de temps en temps on se rend visite " (Nalan, 34 ans, femme au foyer). Le gün est aussi l'occasion de retrouver des voisines que l'on ne voit pas d'une autre façon, comme en témoigne Pelin (65 ans, femme au foyer) à propos de Rezan (36 ans, femme au foyer), qui habite dans l'immeuble d'à côté : «Elle est venue chez moi, moi je n'ai pas pu me rendre chez elle mais nous nous sommes croisées dans beaucoup de gün. » De même, Nuray a fait la connaissance d'Ayşe grâce à leur participation à un jour de réception commun qui leur a donné l'opportunité de se rencontrer à plusieurs reprises : "Nous nous sommes vues plusieurs fois, nous nous voyons dans les gün et autres. » Cependant, contrairement au jour de réception des années 1970 où le réseau était ouvert sans limites, le gün contemporain ressemble plus à un cercle fermé, le réseau ne pouvant s'ouvrir que de proche en proche. Si le gün permet d'élargir son réseau social, c'est en opérant une certaine sélection garantissant la qualité de ses membres :

Nous n'acceptons pas n'importe qui. Par exemple moi, Ayşe hanım est ma proche voisine, on se fréquente, et puis je l'ai recommandée et elles ont accepté. Mais une personne qu'on ne connait pas ne peut pas y participer, ou si c'est quelqu'un qu'on n'apprécie pas on va lui dire qu'on est déjà trop nombreuses ou on trouvera une autre raison pour ne pas l'accepter.

Moyen d'extension du réseau social de chacun, le gün est aussi un important moyen de cohésion de ce réseau. Le gün, contrairement aux relations quotidiennes, comporte une notion d'obligation et de régularité qui crée le lien. Une personne qui ne participerait pas de façon suivie serait, lors de la session suivante, mise à l'écart par les autres participantes ou s'éloignerait de façon spontanée. C'est d'ailleurs une des raisons de la mise en place d'un gün: celui-ci impose une régularité des visites et donne un prétexte incontournable pour se voir. Le fait de se cotiser permet de renforcer le lien moral qui existe entre les participantes du groupe. En effet, si chacune, 
en " entrant dans le gün", s'engage à assister régulièrement aux réunions, la présence de don contraint chacune à s'impliquer davantage, à donner ce qu'elle doit en temps et en heure, et par là à venir assidûment :

Nous faisons un «jour de l'or ", une fois par mois, nous achetons un quart de pièce d'or, et puis ça nous donne une excuse pour nous rendre les unes chez les autres. Nous avons tout d'abord décidé de nous retrouver chez l'une de nous une fois par mois, le cadre de l'école et celui de la maison sont différents. Ensuite elles ont dit que, si on faisait comme ça, il y en a beaucoup qui ne viendraient pas. Nous nous sommes dit que le mieux serait qu'il y ait quelque chose qui nous lie, alors tout le monde viendrait, tout le monde participerait de façon plus régulière. Et puis ça nous arrangeait, parce que c'est une certaine façon d'économiser (Lâle, 30 ans, institutrice).

L'inclusion dans un gün est un engagement moral implicite à y participer avec assiduité durant tout le cycle de visites, sous peine de s'attirer les foudres des autres participantes. Ainsi Halide avait fait la leçon à certaines qui n'avaient pas pu venir chez elle : «Moi, je leur ai dit, nous avons décidé d'un jour précis, vous devez venir, ne serait-ce que pour une demi-heure. Moi, je ne viendrai pas chez vous [...] parce que si on a décidé d'un gün quoi qu'il arrive on doit s'y rendre, même pour une demi-heure. On ne peut pas être absente juste pour le plaisir. » Et, lorsque le jour de réception s'arrête ou si quelqu'un s'en retire, la régularité des relations entre les anciennes participantes diminue comme le déplore Pelin : "L'année dernière, nous avions un gün, [...] un " jour de l'or" avec Nilay, moi, Rezan, la sœur de Rezan, Ayla hanım. Nous nous voyions souvent. Cette année, comme je me suis fait opérer, j'ai arrêté le gün, en sortant du gün je me suis éloignée de mes voisines. "Ayşe fait le même constat : «Avec Dilek hanım, bien sûr nous nous voyons. Avant nous nous voyions une fois par semaine, mais maintenant nous ne pouvons pas nous voir souvent. Pour l'instant notre gün est terminé, nous ne faisons plus de gün, c'est pourquoi nous ne nous voyons pas aussi souvent. "

Le gün est un lieu privilégié d'échange et de circulation d'informations et les liens qui s'y créent sont source de solidarité. Ainsi, au sein d'un gün de voisines, nous avions appris que le fils d'Halide était atteint d'un cancer et, outre le soutien apporté à Halide, il avait été décidé de se rendre ensemble au chevet du malade pour lui souhaiter un prompt rétablissement. Un autre jour, le groupe s'était rendu chez Hilal (34 ans, dessinatrice) qui venait d'accoucher. Celle-ci avait profité de son congé prénatal pour accompagner de façon occasionnelle Halide, sa voisine de palier, au gün organisé dans l'immeuble, ce qui avait renforcé ses liens avec ses autres voisines.

Outre sa dimension sociale, le jour de réception comporte des aspects psychologiques, économiques et religieux. Rassemblant, à sa constitution, des personnes issues d'un même cercle social, il permet une intégration de ses membres et, par ailleurs, d'accéder à d'autres cercles sociaux. Le gün, enfin, grâce à la cohésion de ses membres, autorise, si besoin, leur mobilisation pour une action commune. Dans un contexte de développement urbain, la sociabilité est ainsi passée d'un mode communautaire spontané et quasi quotidien, au sein d'un village ou d'un quartier, à un mode réticulaire, entre membres d'un réseau liés de proche en proche. En formalisant et donnant un cadre à des rencontres auxquelles l'assiduité est obligatoire, le jour de réception permet de créer et, surtout, de maintenir les liens au sein du tissu urbain. Lieu de rires partagés, de larmes séchées, de commensalité, d'échanges d'informations et de richesses, le gün est un moyen de dynamiser un réseau social et de ce fait, reste, malgré l'évolution de la société et l'accroissement du travail des femmes, l'un des piliers de la vie sociale en Turquie urbaine.

\section{Notes}

1. Étymologiquement de l'arabe le « voyageur ". Le terme d'origine turque konuk, "celui qui s'arrête passer une nuit quelque part " est peu utilisé dans la vie sociale ordinaire et se rapporte surtout à des personnes invitées de façon plus formelle comme sur les plateaux de télévision ou lors de visites d'hommes politiques.

2. Voir Benoît Fliche [2006] sur les différences entre les interactions et l'occupation du salon lors des visites quotidiennes ou événementielles.

3. Cependant, à la différence des tontines, le gün ne se focalise pas principalement sur l'argent récolté, dont le montant n'est souvent pas très important, mais qui est plutôt un prétexte pour se réunir.

4. Cette recherche se fonde sur des entretiens et observations conduits à Adana et dans une moindre mesure à Istanbul et Zonguldak. Une longue présence sur le terrain a par ailleurs permis de participer à de nombreux jours de réception dans divers contextes.
5. Je choisis ici de garder le terme turc de gün, sa traduction littérale en français (jour) n'étant pas pertinente et l'expression «jour de réception » renvoyant à une réalité quelque peu différente.

6. De la racine arabe krm qui signifie " généreux ".

7. Voir à ce sujet l'article de Maria Couroucli sur la visite en Grèce [1997].

8. Après l'attentat aux Etats-Unis du 11 septembre 2001 et la chute de la valeur du dollar qui s'en est suivie, la tendance a été de privilégier l'or aux dépens de devises étrangères. 
9. Synonyme d'Almancı, le Turc ayant émigré en Allemagne. Même de retour au pays celui-ci est souvent toujours considéré comme un émigré.
10. Personne qui transmet un enseignement, ici, religieux.

11. Gâteau de semoule très sucré.
12. Halide inclut ici son amie et voisine avec qui elle partage la plupart de ses activités sociales.

\section{Références bibliographiques}

Aswad Barbara C., 1974, "Visiting Pattern among Women of the Elite in a Small Turkish City", Anthropological Quarterly, 47, 1: 9-27.

Barnes John Arundel, 1954, "Class and Committees in a Norwegian Island Parish", Human Relations, 7: 39-58.

BAYARD-ÇAN Hélène, 2008, Voisiner en Turquie urbaine. Une ethnologie de relations réticulaires à Adana, thèse de doctorat de l'Université Paris Ouest Nanterre La Défense.

Benedict Peter, 1974, "The Kabul Günü: Structured Visiting in an Anatolian Provincial Town", Anthropological Quarterly, 47, 1: 28-47.

Couroucli Maria, 1997, «Se rendre chez l'autre : la visite dans la société grecque ", in François Georgeon et Paul Dumont (dir.): Vivre dans l'Empire ottoman: sociabilités et relations intercommunautaires (XVIII $-\mathrm{XX} \mathrm{X}^{e}$ siècles), Paris, Histoire et perspectives méditerranéennes, L'Harmattan : 335-348.

Degenne Alain et Michel Forsé, 1994, Les Réseaux sociaux : une analyse structurale en sociologie, Paris, Armand Colin.

EkAl Berna, 2006, “'How a Kaynana Should Behave?' Discussions on the Role of Mothers-in-Law in Two Gün Groups", European Journal of Turkish Studies, Thematic Issue $\mathrm{N}^{\circ} 4$, The Social Practices of Kinship. A Comparative Perspective. URL : http:// ejts.revues.org/index619.html, consulté en novembre 2012.

FLICHE Benoît, 2006, "L'événement et le quotidien. Une ethnologie du salon dans un quartier de gecekondu d'Ankara (Turquie) ", Anthropology of the Middle East, 1, 1:51-62.

Grossettr Michel, 2005, "Where Do Social Relations Come From? A Study of Personal Networks in the Toulouse Area of France", Social Networks, 27 : 289-300.

Khatı-Chahidi Jane, 1995, "Gold Coins and Coffee ROSCAs: Coping with Inflation the Turkish Way in Northern
Cyprus", in Shirley Ardener, Sandra Burman (eds.), Money go Rounds, the Importance of Rotating Savings and Credit Associations for Women, Oxford, Berg: 241-261.

KIray Mubeccel, 1981, "The Women of Small Town”, in Nermin Abadan-Unat (ed.), Women in Turkish Society, Leiden, E.J. Brill: 259-274.

Mauss Marcel, 1993, «Essai sur le don. Formes et raisons de l'échange dans les sociétés archaïques ", in Sociologie et anthropologie, Paris, Presses universitaires de France : 143-279.

SAuner Marie-Hélène, 2001, "Espaces et réseaux de femmes migrantes à Istanbul ", Anatolia Moderna, X : 212-217.

Sonmez Gulsun A., Metin Argan, Turkan N. SabirLI and Tuba SEvil, 2010, "A Different Leisure Activity for Turkish Women: Invitation Day", World Leisure Journal, 52, 2: 94-103. URL : http://dx.doi.org/10.1080/04419057.2010.9674634, consulté en novembre 2012.

TAPper Nancy, 1990, "Ziyaret: Gender, Movement, and Exchange in a Turkish Community", in Dale. F. Eickelman, James Piscatori (eds): Muslims, Travellers: Pilgrimage, Migration and the Religious Imagination, Londres, Routledge: 236-255.

Wellman Barry, 1979, "Networks, Neighborhoods, and Communities: Approaches to the Study of the Community Question ", Urban Affairs Quarterly, 14, 3 : 363-390.

Wolbert Barbara, 1992, “Günümüz - 'unser Tag' : Skizze eines Frauentreffens in Izmir mit Bemerkungen zu einer Neuen Form des Kabul Günü in der Urbanen Türkei" [ "Notre jour » : Esquisse d'une réunion féminine à Izmir avec des remarques sur une nouvelle forme de jour de réception en Turquie urbaine], Anthropos, 87 : 214-223.

Wolbert Barbara, 1996, "The Reception Day - A Key to Migrant's Reintegration”, in Gabriele Rasuly-Paleczek (ed.): Turkish Families in Transition, Frankfurt am Main-Berlin-Bern-New York-Paris-Wien, Peter Lang: 186-215. 


\section{ABSTRACT}

The reception day in urban Turkey. Social networks and sociability

As visits take an important place within the Turkish sociability, reception days (gün) are an institution. This paper, based on interviews and observations conducted in several Turkish cities, studies the characteristics of this mode of sociability. After studying its social, psychological, economic and religious aspects, this paper focuses on its reticular dimension and shows its importance in the constitution, extension and mobilisation of everyone's social network.

Keywords : Sociability. Gün. Reception day. Social network. Turkey

\section{ZUZAMMENFASSUNG}

Empfangstag in der städtischen Türkei. Soziale Netzwerke und Geselligkeit

Besuche sind untrennbar mit der türkischen Auffassung von Geselligkeit verbunden und Empfangstag (gün) sind hierbei eine Art Institution. Der Beitrag untersucht diese Art der Geselligkeit anhand von Befragungen und Beobachtungen in mehreren türkischen Städten. Der Autor betrachtet die soziale, psychologische, wirtschaftliche und religiöse Dimension des Ritus und zeigt seine Wichtigkeit für den Aufbau, den Fortbestand und die Lebendigkeit persönlicher sozialer Netzwerke auf.

Stichwörter: Geselligkeit. Gün. Willkommensritus. Soziales Netzwerk. Türkei.

\section{| ÖZET}

Kentsel Türkiye'de gün toplantıları. Sosyal ağları ve sosyabilite

Türkiye'de, misafir ziyaretleri, sosyal ilişkiler açısından önemli bir yer almakla birlikte, gün ziyaretleri daha kurumsal bir konumdadır. Bu makalede, günlere ilişkin sosyal faaliyet özellikleri, Türkiye'nin değişik şehirlerinde yapılmış anket ve gözlemler dikkate alınarak değerlendirilmiştir. İlk olarak sosyallik, psikolojik, ekonomik ve dinsel boyutlar değerlendirildikten sonra, sosyal ağ açısından, bireylerin ağın oluşumunda, genişlemesinde ve harekete geçmesinde önemi vurgulanmıştır.

Anahtar kelimeler : Sosyallik. Gün. Kabul günü. Sosyal ăg. Türkiye. 